\title{
MUSINGS
}

\section{Hunting for cancer in the microbial jungle}

\author{
Pauline Funchain ${ }^{1,2}$ and Charis Eng ${ }^{1,2,3,4,5 *}$
}

In a time when the fragility of the global ecosystem is a hot topic, the macrosystem of global environmental problems parallels the microcosm of the biomedical sphere known as the human body. We are beginning to understand that the safari of a world that inhabits our bodies is highly diverse and sensitive to host-environment factors, and that perturbations of this jungle mirror, and perhaps cause, diseases as disparate as inflammatory bowel disease, diabetes, and obesity [1-3].

Medically, we have understood the concept of perturbing the human ecosystem for some time. As with monocultures in agriculture, razing the landscape of human prosymbionts has serious medical consequences, usually resulting from re-colonization by a predominant species that was previously held in check by the overall community. For example, courses of antibiotics for urinary tract infections are known triggers of vaginal yeast infections; equally, antibiotics for bacterial pneumonias and bloodstream infections are associated with the ever problematic Clostridium difficile diarrhea. The supposition that disturbing the microenvironment causes disease seems to be supported by the recent proof-of-principle experiment to treat disease by repopulating a devastated ecosystem with a healthy one, resulting in the successful eradication of $C$. difficile colitis through fecal microbial transplantation [4].

\section{Diversity in the microbial habitat: why is more better?}

Evidence is mounting that microbial diversity is healthy and less diversity is not. A seminal study in lean and obese twins [3] showed that less diversity in the gut microbiome correlated with obesity, and multiple studies of other diseases, such as inflammatory bowel disease and colon cancer [1,5], consistently find that lower diversity occurs in disease states. Repeatedly finding less diversity in multiple, seemingly separate diseases is probably not a coincidence.

\footnotetext{
*Correspondence: engc@ccf.org

'Genomic Medicine Institute, Cleveland Clinic, 9500 Euclid Avenue, NE-50,

Cleveland, Ohio 44195, USA

Full list of author information is available at the end of the article
}

Consider the finding that microbiomes of cancerous tissue show lower diversity than adjacent normal tissue, recently reported for colorectal cancer [5], and consider how disturbing the microcosm by reducing its diversity might produce a malignant state. One possibility is that shifting the microcosm such that a few species predominate might allow normally benign organisms to become pathogenic as a result of overrepresentation, as in the case of $C$. difficile. Such microbial dysbiosis could potentially explain the differences between colonization and pathogenesis of Helicobacter pylori in the case of gastric cancer, or human papilloma virus on squamous cell surfaces of the cervix, anus, or head and neck. A second possibility is that a different landscape of microorganisms would result in a different ecosystem of metabolites. Perhaps a decrease in diversity of microorganisms leads to a decrease in diversity of metabolites, creating a 'serum starvation' condition, that is, a state in which essential nutrients are lacking. In such a state, an increase in selective pressures might favor hyperproliferative clones and thus predispose to cancer. Alternatively, shifts in diversity might allow an oncometabolite-producing organism, otherwise benign in small proportions, to flourish and produce metabolites that are carcinogenic in large quantities. For example, although ethanol itself is not carcinogenic, its metabolite acetaldehyde, converted from ethanol in vivo by both bacteria and fungi (particularly Candida albicans), is carcinogenic in animal models [6]. A third possibility is that a decrease in diversity could cause a shift in the body's baseline immunological response. One imagines either an overactivation of the immune system causing inflammatory changes such as global hypermethylation [7] or a quiescence of the immune system allowing cancer to grow.

\section{Profiling cancers by their microbiomes: where the wild things are}

Microbial profiling of cancer is agnostic as to whether bacteria are the cause or the effect, although in each scenario lies clinical utility. If the development of cancer is the underlying reason for shifts in the microbial community, then surveying bacteria becomes a new method for diagnosis. Microbial censuses could be used to diagnose malignancies and in surveillance for 
recurrence. Of clinical importance is that microbiome sampling, as opposed to blood sampling or tissue biopsies, is generally non-invasive. Such approaches are already revealing insights; for example, specific microbes in saliva have been associated with chronic pancreatitis and tumors of the pancreas [8]. On the other hand, if shifts in the microbial spectrum cause cancer, then not only does microbiome research become a new route into exploring pathogenesis but the microbiome itself becomes a new target for preventative strategies. Microbiomes could be sampled proactively for risk stratification in at-risk populations, and probiotics and microbiome transplants could be considered as strategies for cancer prevention.

Also intriguing is the idea that one might be able to categorize cancers that do or do not have a microbial influence. It has been demonstrated in head and neck cancers that microbes live deep within tumor tissue [9], thus contributing to the intracellular stromal environment. Unless their borders are breached by infection, organs such as the kidney and the pancreas have been thought to be completely sterile. With modern metagenomic profiling, which can identify fastidious and previously unculturable microorganisms, we might find that fewer organ systems are sterile than previously thought, and that the contribution of the microbial microenvironment might have a much more inwardreaching influence than currently assumed. Classifying cancers into sterile and non-sterile, if sterile cancers truly exist, might garner increased biological understanding of tumorigenesis, perhaps providing new avenues for diagnosis and even novel treatment modalities for nonsterile cancers.

In the jungle, an acute sense of smell, an awareness of changes in the pattern of bushes moving, and a sensitivity to the sounds and vibrations of a herd far away can help a hunter smoke out a nearby predator or prey. If we view each human organ, and probably subareas of these organs, as a separate ecosystem of symbiotic microbes and human 'terrain', we can better appreciate the interplay of native species within their environmental niche. If we understand cancer to be a predator causing shifts in this delicately balanced jungle, paying attention to the environmental cues dropped by our microbial wildlife might not only broaden our understanding of the ecological community within the jungle but might also help us to refine our cancer-hunting senses.

\section{Acknowledgements}

$\mathrm{PF}$ is the Ambrose Monell Foundation Cancer Genomic Medicine Clinical Fellow at the Cleveland Clinic Genomic Medicine Institute. CE is the Sondra $J$ and Stephen R Hardis Chair of Cancer Genomic Medicine at the Cleveland Clinic and is an American Cancer Society Clinical Research Professor,

generously funded in part by the FM Kirby Foundation.

\section{Competing interests}

The authors declare that they have no competing interests.

\section{Author details}

'Genomic Medicine Institute, Cleveland Clinic, 9500 Euclid Avenue, NE-50, Cleveland, Ohio 44195, USA. ²Lerner Research Institute, Cleveland Clinic, Cleveland, Ohio 44195, USA. ${ }^{3}$ Taussig Cancer Institute, Cleveland Clinic, Cleveland, Ohio 44195, USA. ${ }^{4}$ Stanley Shalom Zielony Institute of Nursing Excellence, Cleveland Clinic, Cleveland, Ohio 44195, USA. ${ }^{5}$ Department of Genetics and Genome Sciences, and CASE Comprehensive Cancer Center, Case Western Reserve University, Cleveland, Ohio 44106, USA.

Published: 29 May 2013

\section{References}

1. Manichanh C, Rigottier-Gois L, Bonnaud E, Gloux K, Pelletier E, Frangeul L, Nalin R, Jarrin C, Chardon P, Marteau P: Reduced diversity of faecal microbiota in Crohn's disease revealed by a metagenomic approach. Gut 2006, 55:205-211.

2. Qin J, Li Y, Cai Z, Li S, Zhu J, Zhang F, Liang S, Zhang W, Guan Y, Shen D, Peng Y, Zhang D, Jie Z, Wu W, Qin Y, Xue W, Li J, Han L, Lu D, Wu P, Dai Y, Sun X, Li Z, Tang A, Zhong S, Li X, Chen W, Xu R, Wang M, Feng Q, et al:: A metagenomewide association study of gut microbiota in type 2 diabetes. Nature 2012, 490:55-60.

3. Turnbaugh PJ, Hamady M, Yatsunenko T, Cantarel BL, Duncan A, Ley RE, Sogin ML, Jones WJ, Roe BA, Affourtit JP, Egholm M, Henrissat B, Heath AC, Knight R, Gordon Jl: A core gut microbiome in obese and lean twins. Nature 2009, 457:480-484.

4. van Nood E, Vrieze A, Nieuwdorp M, Fuentes S, Zoetendal EG, de Vos WM, Visser CE, Kuijper EJ, Bartelsman JFWM, Tijssen JGP, Speelman P, Dijkgraaf MGW, Keller JJ: Duodenal infusion of donor feces for recurrent Clostridium difficile. N Engl J Med 2013, 368:407-415.

5. Chen W, Liu F, Ling Z, Tong $X$, Xiang $C$ : Human intestinal lumen and mucosaassociated microbiota in patients with colorectal cancer. PloS One 2012, 7:e39743.

6. Hooper SJ, Wilson MJ, Crean SJ: Exploring the link between microorganisms and oral cancer: a systematic review of the literature. Head Neck 2009, 31:1228-1239.

7. Bebek G, Bennett KL, Funchain P, Campbell R, Seth R, Scharpf J, Burkey B, Eng C: Microbiomic subprofiles and MDR1 promoter methylation in head and neck squamous cell carcinoma. Hum Mol Genet 2012, 21:1557-1565.

8. Farrell J, Zhang L, Zhou H, Chia D, Elashoff D, Akin D, Paster BJ, Joshipura K, Wong DT: Variations of oral microbiota are associated with pancreatic diseases including pancreatic cancer. Gut 2011, 61:582-588.

9. Hooper SJ, Crean SJ, Fardy MJ, Lewis MAO, Spratt DA, Wade WG, Wilson MJ: A molecular analysis of the bacteria present within oral squamous cell carcinoma. J Med Microbiol 2007, 56:1651-1659.

doi:10.1186/gm446

Cite this article as: Funchain P, Eng C: Hunting for cancer in the microbial jungle. Genome Medicine 2013, 5:42. 